\title{
A Primary Barrett's Adenocarcinoma with a Squamous Cell Carcinoma Component
}

\author{
Akio Takeuchi ${ }^{1}$, Waku Hatta ${ }^{1}$, Tomoyuki Koike ${ }^{1}$, Masahiro Saito ${ }^{1}$, Xiaoyi Jin ${ }^{1}$, \\ Kiyotaka Asanuma ${ }^{1}$, Kaname Uno ${ }^{1}$, Naoki Asano ${ }^{1}$, Akira Imatani ${ }^{1}$, \\ Fumiyoshi Fujishima $^{2}$ and Atsushi Masamune ${ }^{1}$
}

\begin{abstract}
:
The present report describes an extremely rare case of Barrett's adenocarcinoma (BAC) with a squamous cell carcinoma (SCC) component. A 55-year-old man was diagnosed with esophageal adenocarcinoma on Barrett's esophagus. The patient underwent endoscopic submucosal dissection, but the pathology revealed deep submucosal invasive, moderately differentiated tubular adenocarcinoma and focal SCC with vascular invasion. In addition, morphological transition between adenocarcinoma and SCC components was confirmed. The patient underwent additional surgery, which revealed lymph node metastasis, and then received S-1 adjuvant chemotherapy. Based on the pathological findings, the transdifferentiation process may have a role in the histogenesis of this tumor.
\end{abstract}

Key words: Barrett's adenocarcinoma, squamous cell carcinoma component, morphological transition

(Intern Med 58: 2467-2472, 2019)

(DOI: 10.2169/internalmedicine.2722-19)

\section{Introduction}

In Western countries, the rate of esophageal adenocarcinoma, most cases of which arise in a Barrett's esophagus, has been increasing for the past few decades, accounting for approximately $60 \%$ of esophageal cancer cases; however, in Japan, squamous cell carcinoma (SCC) occurs in most esophageal cancer patients, and the incidence of esophageal adenocarcinoma remains below $10 \%$ (1). Therefore, in Japan, Barrett's adenocarcinoma (BAC) has remained a relatively rare neoplasm. Since most Barrett's cancers are adenocarcinomas that develop through the esophagitismetaplasia-dysplasia-carcinoma sequence, esophageal neoplasms that contain elements of SCC and adenocarcinoma in a background of Barrett's esophagus are extremely rare.

We herein report a rare case of BAC with an SCC component.

\section{Case Report}

A 55-year-old man without any symptoms underwent an X-ray barium examination as part of an annual medical checkup, and an irregular indentation was identified at the esophagogastric junction. Esophagogastroduodenoscopy (EGD) at a hospital indicated a depressed lesion on Barrett's esophagus. A biopsy specimen taken from the lesion revealed well-differentiated adenocarcinoma. The patient was then referred to our institution for a further examination and treatment of the esophageal lesion.

The patient showed no relevant medical history. He had a habit of smoking (1 pack/day, 35 years) and drinking 350 $\mathrm{mL}$ of beer and $180 \mathrm{~mL}$ of shochu daily, containing $50 \mathrm{~g}$ of ethanol. A clinical examination and laboratory studies showed unremarkable results. EGD repeated at our institution revealed a whitish, depressed lesion with an elevation of its epicenter (Fig. 1a and b) on Barrett's esophagus, which was classified as C3M6 according to the Prague C \&

${ }^{1}$ Division of Gastroenterology, Tohoku University Graduate School of Medicine, Japan and ${ }^{2}$ Department of Pathology, Tohoku University Graduate School of Medicine, Japan

Received: January 21, 2019; Accepted: March 1, 2019; Advance Publication by J-STAGE: May 22, 2019

Correspondence to Dr. Waku Hatta, waku-style@ festa.ocn.ne.jp 


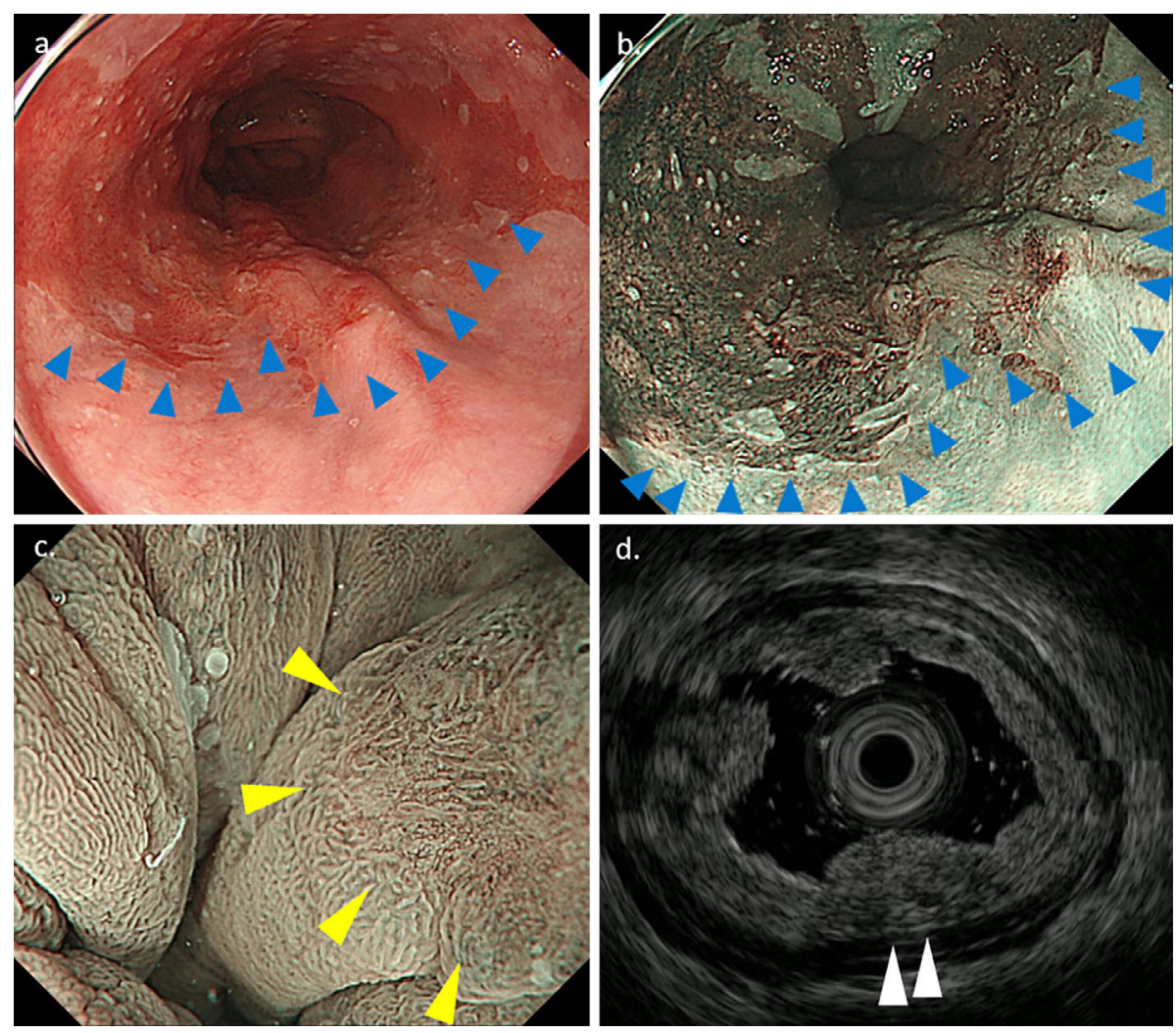

Figure 1. Endoscopic images of a case that has a BAC with a SCC component. White-light imaging showed a whitish, depressed lesion with an elevated epicenter on Barrett's esophagus and findings suggestive of subsquamous carcinoma extension at the oral side of the lesion (a). NBI identified this tumor as a brownish area (b). The blue arrows indicate the oral margin of the tumor $(\mathbf{a}, \mathbf{b})$. The anal margin of the tumor was clearly demarcated on magnifying endoscopy with NBI (yellow arrow) (c). EUS showed a hypoechoic mass and irregular narrowing of sonographic layer 3 (white arrow) (d), diagnosed as deep submucosal invasion. BAC: Barrett's adenocarcinoma, SCC: squamous cell carcinoma, NBI: narrow-band imaging, EUS: endoscopic ultrasonography

M criteria (2). The oral side of the elevated region was covered with squamous epithelium, suggesting subsquamous carcinoma extension. The anal margin of this tumor was clearly demarcated on magnifying endoscopy with narrowband imaging (NBI) (Fig. 1c). Endoscopic ultrasonography (EUS) showed a hypoechoic mass and irregular narrowing of the third sonographic layer (Fig. 1d), which was diagnosed as deep submucosal invasion. There was no evidence of lymph nodes or distant metastasis on computed tomography or positron emission tomography. Based on these findings, the preoperative diagnosis of this tumor was Barrett's adenocarcinoma with submucosal invasion (T1b, N0, M0, stage IB). According to the Japanese guidelines for the diagnosis and treatment of carcinoma of the esophagus (3), radical surgery was recommended for the patient. However, he selected endoscopic resection as the initial treatment method.

The patient underwent endoscopic submucosal dissection (ESD) without any major complications. The pathology revealed deep submucosal invasion $(2,090 \mu \mathrm{m}$ from the deep muscularis mucosa), moderately differentiated tubular adenocarcinoma and focal SCC with venous invasion but no lymphatic invasion and a maximal size of $4.5 \mathrm{~cm}$. The horizon- tal margin was negative, but the vertical margin was unclear. Barrett's mucosa was identified at the anal side of the tumor (Fig. 2). The SCC component occupied only about $10 \%$ of the lesion (Fig. 3), and morphological transition between adenocarcinoma and SCC components was confirmed by Hematoxylin and Eosin ( $\mathrm{H} \& \mathrm{E})$ staining and immunostaining (Fig. 4). In addition, all of the SCC component was covered by the adenocarcinoma component (Fig. 5). According to the guidelines for clinical and pathological studies of carcinomas of the esophagus established by the Japan Esophageal Society (4), the final pathological diagnosis of the tumor was BAC with an SCC component. Because of the risk of lymph node metastasis (LNM) and residual tumor, additional radical surgery was strongly recommended for the patient, and thoracoscopic esophagectomy followed by gastric tube reconstruction was performed. The resected specimen showed no residual tumor; however, 2/15 lymph nodes contained metastases of adenocarcinoma. The pathological staging of this tumor was therefore T1b, N1, M0, stage IIB. Subsequently, the patient received S-1 adjuvant chemotherapy. No recurrence was noted during 12 months of followup. 

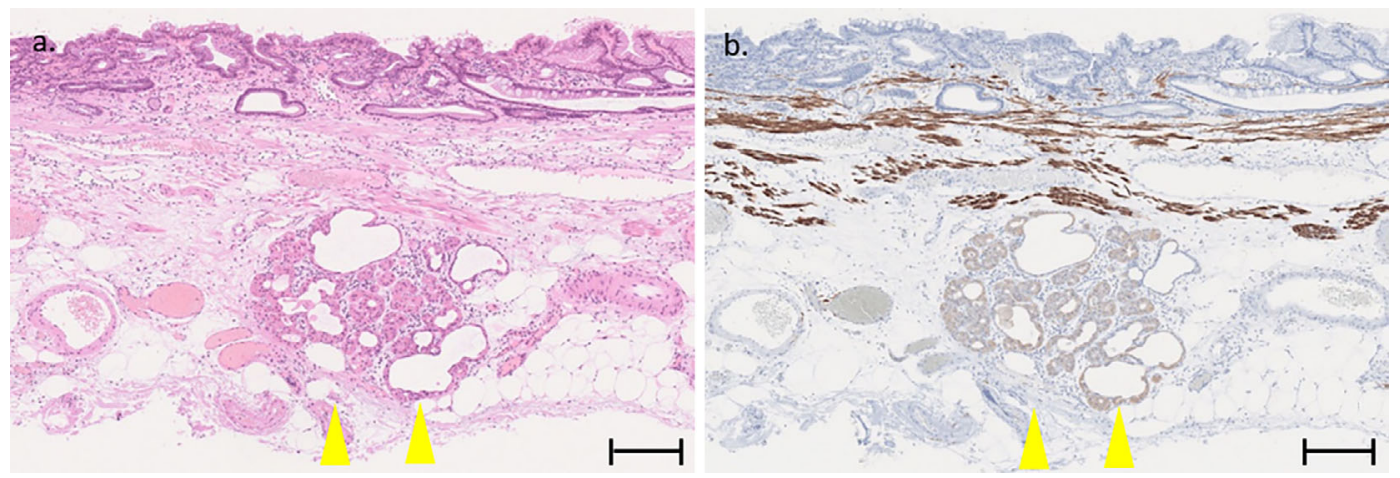

Figure 2. Barrett's esophagus. At the anal side of the tumor, the pathology showed duplication of the muscularis mucosa and esophageal glands (yellow arrow) on Hematoxylin and Eosin staining (a) and desmin staining (b), corresponding to Barrett's esophagus. Bar=200 $\mu \mathrm{m}$
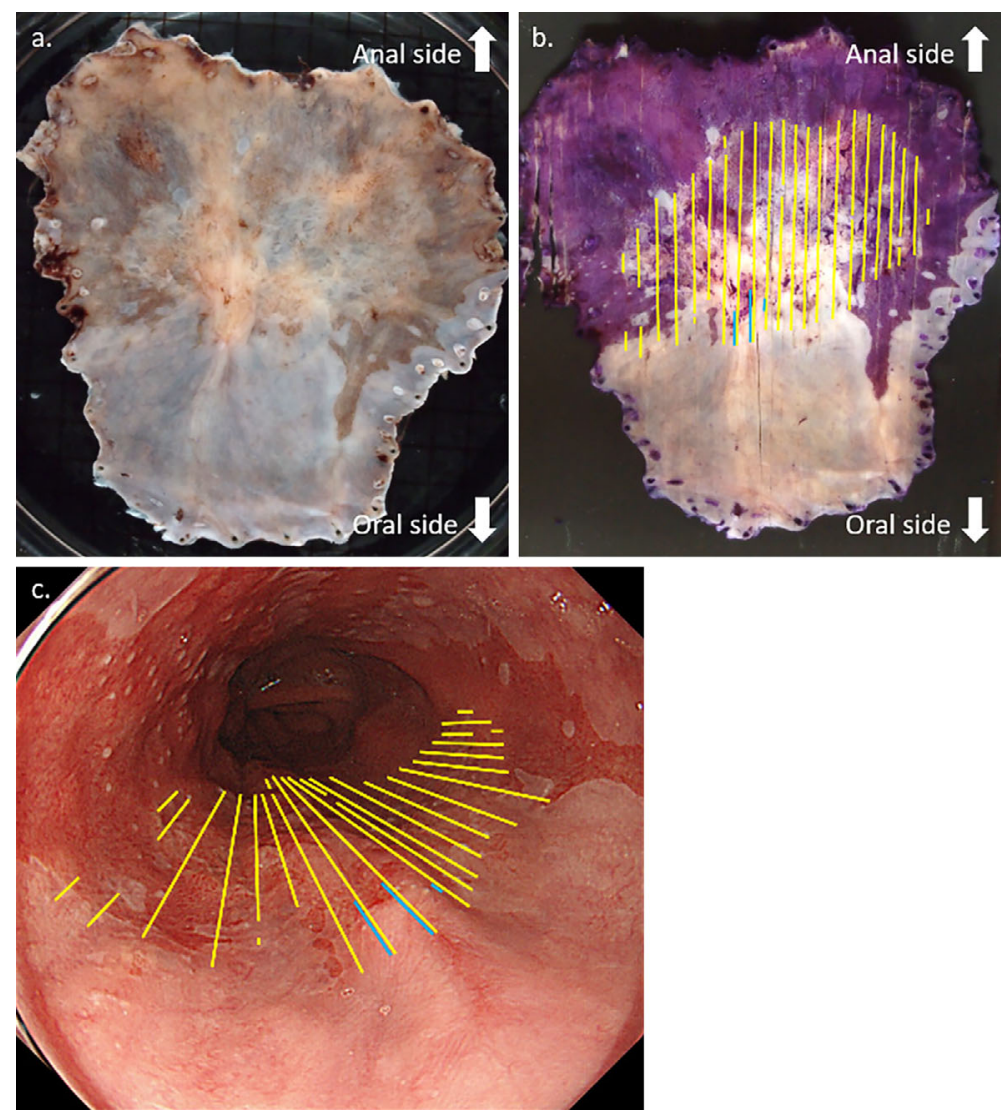

Figure 3. ESD specimen and reconstructive images based on the pathology in the specimen stained by Hematoxylin and Eosin staining and endoscopy. A whitish lesion was shown in the resected specimen (a). Adenocarcinoma (yellow lines) appeared in the whole area of the lesion, but SCC (blue lines) occupied only about $10 \%$ of the lesion (b, c). ESD: endoscopic submucosal dissection, SCC: squamous cell carcinoma

\section{Discussion}

Esophageal adenosquamous carcinoma is a rare tumor, accounting for only $0.37-1.0 \%$ of all esophageal cancers (5). In addition, carcinoma containing coexisting elements of infiltrating adenocarcinoma and SCC in the background of Barrett's mucosa is extremely rare. Thus far, only six cases of BAC containing elements of SCC have been reported (Table) (6-11), making the present case the seventh case of such carcinoma. According to the guidelines for clinical and pathological studies of carcinomas of the esophagus established by the Japan Esophageal Society (4), adenosquamous carcinoma of the esophagus is defined as a lesion comprising $\geq 20 \%$ of SCC and adenocarcinoma features on a routine microscopic examination using $\mathrm{H} \& \mathrm{E}$ staining. However, 

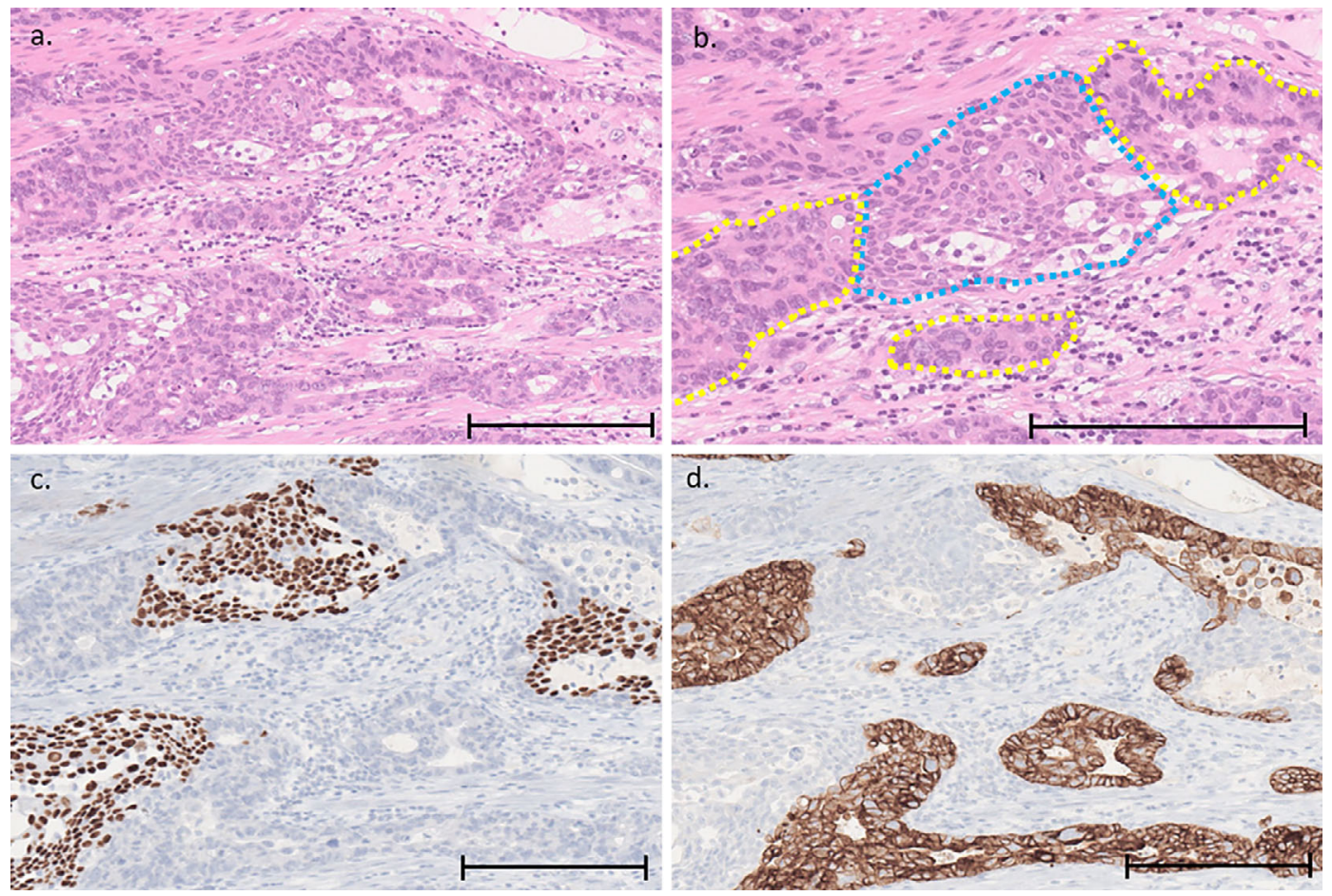

Figure 4. Morphological transition between adenocarcinoma and SCC. Morphological transition between adenocarcinoma and SCC was demonstrated by Hematoxylin and Eosin staining (a, b) and immunostaining [with P40 (c), a marker for squamous epithelial cells, and CK18 (d), a marker for columnar epithelial cells]. In the high-magnification image (b), the yellow-dotted areas correspond to the adenocarcinoma component, whereas the blue-dotted area corresponds to the SCC component. SCC: squamous cell carcinoma, Bar $=200 \mu \mathrm{m}$

the WHO classification defines adenosquamous carcinoma as having elements of both SCC and adenocarcinoma without providing a reference concerning the ratio of these components (12). The present case mainly consisted of an adenocarcinoma component, with the SCC component representing about $10 \%$ of the lesion. It was therefore defined as BAC with an SCC component according to the Japanese classification and Barrett's adenosquamous carcinoma according to the WHO classification. Thus far, only one other case of BAC with an SCC component has been reported (11). However, that case report did not mention the ratio of the SCC component, and based on the reconstructed pictures of the report, the tumor appears to have been over $20 \%$ SCC. Therefore, this is the first case with a precise diagnosis of BAC with a SCC component. The presence of such a small SCC component in the lesion may be key to understanding the histogenesis of BAC containing elements of SCC.

Two possible histogeneses of coexisting elements of adenocarcinoma and SCC have been proposed. One involves the collision between adenocarcinoma and SCC. Previous studies hypothesized that two individual stem cells may have simultaneously and independently undergone malignant transformation $(5,13-15)$, and another report suggested that SCC in Barrett's esophagus may have arisen from an islet of squamous epithelium in Barrett's esophagus (16). The other potential suggested histogenesis involves monoclonal origin.
In their molecular analysis, van Rees et al. showed that both the adenocarcinoma and SCC components originated from Barrett's mucosa that surrounded the cancer, and the divergence of these components presumably occurred as a late event of histogenesis (9). Although we did not conduct a molecular analysis in the present study, a morphological transition between adenocarcinoma and SCC was clearly shown in this case. In addition, the SCC component did not appear on the surface of the tumor, and the whole SCC component in the lamina propria mucosa and muscularis mucosa was covered by the adenocarcinoma component. These findings support the histogenesis suggested by van Rees et al. The tumor in our case likely first developed as BAC, and then SCC differentiation occurred within the tumor cell populations after adenocarcinoma invaded the lamina propria mucosa.

No cases treated by endoscopic resection for cancers containing both elements of adenocarcinoma and SCC in the background of Barrett's mucosa have yet been reported. Although the present patient underwent ESD for his tumor, additional radical surgery was required, which revealed LNM. These findings suggest that it may be difficult to curatively treat esophageal cancers containing both elements of adenocarcinoma and SCC by endoscopic resection. In addition, based on the present and previous findings, the rate of LNM in T1 BAC containing elements of SCC appears to be $75 \%$ (3/4), which is much higher than that in T1b BAC (17.5- 

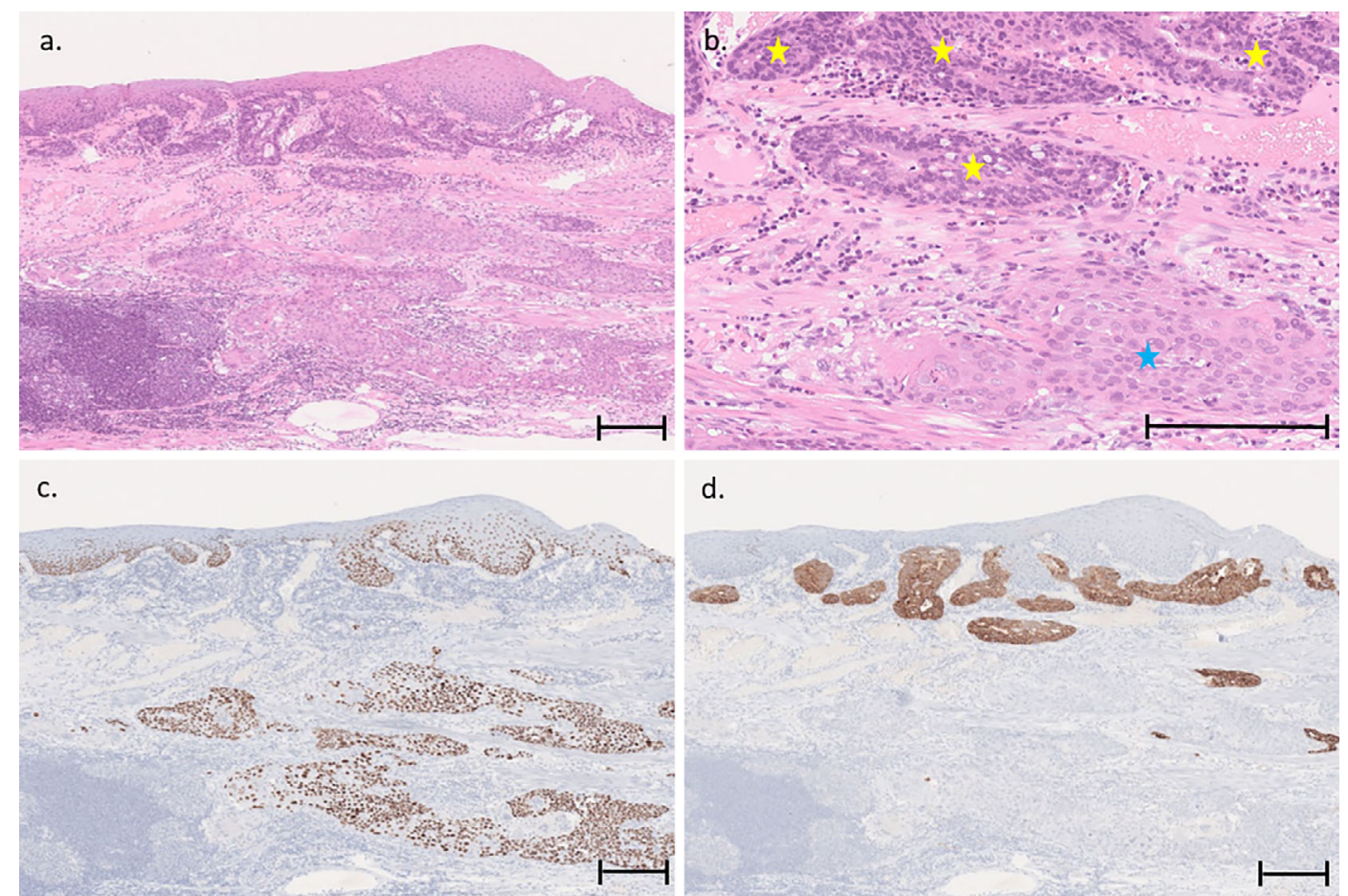

d.

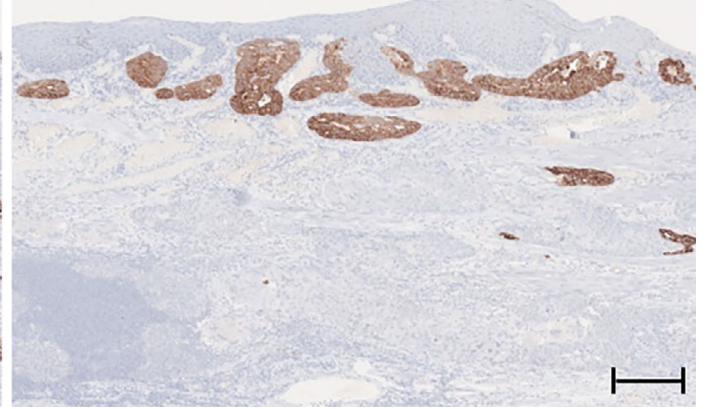

Figure 5. SCC component covered by adenocarcinoma. In this tumor, the entire SCC component was covered by adenocarcinoma [Hematoxylin and Eosin (H\&E) staining (a, b), P40 (c), a marker for squamous epithelial cells, and CK18 [d], a marker for columnar epithelial cells]. The adenocarcinoma extended underneath the normal squamous epithelium (a, c, d). In the high-magnification image of H\&E staining (b), the blue star corresponds to the SCC component, whereas the yellow stars correspond to the adenocarcinoma component. SCC: squamous cell carcinoma, Bar=200 $\mu \mathrm{m}$

Table. Reported Cases of BAC That Contains Elements of SCC.

\begin{tabular}{|c|c|c|c|c|c|c|c|c|}
\hline Case & $\begin{array}{c}\text { Age } \\
\text { (years)/ } \\
\text { Sex }\end{array}$ & $\begin{array}{c}\text { Type of } \\
\text { Barrett's } \\
\text { esophagus }\end{array}$ & $\begin{array}{l}\text { Tumor } \\
\text { size } \\
(\mathrm{cm})\end{array}$ & $\begin{array}{l}\text { Macroscopic } \\
\text { morphology }\end{array}$ & Therapy & $\begin{array}{c}\text { TNM } \\
\text { classification }\end{array}$ & $\begin{array}{l}\text { Alive } \\
\text { or dead }\end{array}$ & $\begin{array}{c}\text { Follow-up } \\
\text { duration } \\
\text { (month) }\end{array}$ \\
\hline Ref. 6) & $50 /$ male & - & 6 & Flat & Surgery & $\begin{array}{l}\text { T3NOM0 } \\
\text { Stage IIB }\end{array}$ & Dead & 9 \\
\hline Ref. 7) & $63 / \mathrm{male}$ & LSBE & 3 & $\begin{array}{c}\text { Centrally } \\
\text { ulcerated mass }\end{array}$ & Surgery+RT & - & Dead & 4 \\
\hline Ref. 8) & $85 /$ male & - & - & $\begin{array}{l}\text { Submucosal } \\
\text { mass }\end{array}$ & Surgery & $\begin{array}{l}\text { T3N1M0 } \\
\text { Stage IIIB }\end{array}$ & - & - \\
\hline Ref. 9) & $72 /$ male & LSBE & 3 & Ulceration & Surgery & $\begin{array}{l}\text { T1bN1M0 } \\
\text { Stage IIB }\end{array}$ & - & - \\
\hline Ref. 10) & $72 /$ male & LSBE & 5 & $\begin{array}{l}\text { Localized } \\
\text { ulceration }\end{array}$ & Surgery & $\begin{array}{l}\text { T1bN1M0 } \\
\text { Stage IIB }\end{array}$ & Dead & 17 \\
\hline Ref. 11) & $52 /$ male & LSBE & 3.5 & 0-IIa+IIc & Surgery & $\begin{array}{l}\text { T1bN0M0 } \\
\text { Stage IB }\end{array}$ & Alive & 24 \\
\hline Present case & $55 /$ male & LSBE & 4.5 & 0-IIc+IIa & $\begin{array}{c}\text { ESD } \rightarrow \\
\text { Surgery+Chemotherapy }\end{array}$ & $\begin{array}{l}\text { T1bN1M0 } \\
\text { Stage IIB }\end{array}$ & Alive & 12 \\
\hline
\end{tabular}

BAC: Barrett's adenocarcinoma, SCC: squamous cell carcinoma, LSBE: long-segment Barrett's esophagus, RT: radiation therapy, ESD: endoscopic submucosal dissection

$31.0 \%)(17-21)$. Although the small number of cases prevents us from drawing any definitive conclusions, BAC containing elements of SCC may have a more aggressive behavior than BAC.

We herein report an extremely rare case of BAC with an SCC component that showed morphological transition be- tween adenocarcinoma and SCC. The present findings suggest that a transdifferentiation process may play a role in the histogenesis of this tumor.

The authors state that they have no Conflict of Interest (COI). 


\section{References}

1. Nishi T, Makuuchi H, Ozawa S, Shimada H, Chino O. The present status and future of Barrett's esophageal adenocarcinoma in Japan. Digestion 99: 185-190, 2019.

2. Sharma P, Dent J, Armstrong D, et al. The development and validation of an endoscopic grading system for Barrett's esophagus: the Prague C \& M criteria. Gastroenterology 131: 1392-1399, 2006.

3. Kuwano H, Nishimura Y, Oyama T, et al. Guidelines for diagnosis and treatment of carcinoma of the esophagus April 2012 edited by the Japan Esophageal Society. Esophagus 12: 1-30, 2015.

4. Japan Esophageal Society. Japanese classification of esophageal cancer, 11th Edition: part II and III. Esophagus 14: 37-65, 2017.

5. Zhang HD, Chen CG, Gao YY, et al. Primary esophageal adenosquamous carcinoma: a retrospective analysis of 24 cases. Dis Esophagus 27: 783-789, 2014.

6. Smith RR, Hamilton SR, Boitnott JK, Rogers EL. The spectrum of carcinoma arising in Barrett's esophagus. A clinicopathologic study of 26 patients. Am J Surg Pathol 8: 563-573, 1984.

7. Pascal RR, Clearfield HR. Mucoepidermoid (adenosquamous) carcinoma arising in Barrett's esophagus. Dig Dis Sci 32: 428-432, 1987.

8. Ter RB, Govil YK, Leite L, et al. Adenosquamous carcinoma in Barrett's esophagus presenting as pseudoachalasia. Am J Gastroenterol 94: 268-270, 1999.

9. van Rees BP, Rouse RW, de Wit MJ, et al. Molecular evidence for the same clonal origin of both components of an adenosquamous Barrett carcinoma. Gastroenterology 122: 784-788, 2002.

10. Noguchi $T$, Uchida $Y$, Fumoto $S$, Wada $S$, Sato $T$, Takeno $S$. Adenosquamous carcinoma arising in Barrett's esophagus. Jpn J Thorac Cardiovasc Surg 50: 537-540, 2002.

11. Mishima Y, Amano Y, Yuki T, et al. A rare case of Barrett's adenocarcinoma including squamous cell carcinoma component. Clin J Gastroenterol 4: 5-9, 2011.

12. Flejou JF. [WHO Classification of digestive tumors: the fourth edition]. Ann Pathol 31 (5 Suppl): S27-S31, 2011 (in French).

13. Epstein JI, Sears DL, Tucker RS, Eagan JW Jr. Carcinoma of the esophagus with adenoid cystic differentiation. Cancer 53: 11311136, 1984.

14. Sakata K, Ishida M, Hiraishi $\mathrm{H}$, et al. Adenosquamous carcinoma of the esophagus after endoscopic variceal sclerotherapy: a case report and review of the literature. Gastrointest Endosc 47: 294299, 1998.

15. Keller JJ, Westerman AM, de Rooij FW, et al. Molecular genetic evidence of an association between nasal polyposis and the PeutzJeghers syndrome. Ann Intern Med 136: 855-856, 2002.

16. Streppel MM, Siersema PD, de Leng WW, et al. Squamous cell carcinoma in Barrett's esophagus: field effect versus metastasis. Dis Esophagus 25: 630-637, 2012.

17. Stein HJ, Feith M, Bruecher BL, Naehrig J, Sarbia M, Siewert JR. Early esophageal cancer: pattern of lymphatic spread and prognostic factors for long-term survival after surgical resection. Ann Surg 242: 566-573, 2005.

18. Altorki NK, Lee PC, Liss Y, et al. Multifocal neoplasia and nodal metastases in T1 esophageal carcinoma: implications for endoscopic treatment. Ann Surg 247: 434-439, 2008.

19. Pennathur A, Farkas A, Krasinskas AM, et al. Esophagectomy for T1 esophageal cancer: outcomes in 100 patients and implications for endoscopic therapy. Ann Thorac Surg 87: 1048-1054, 2009.

20. Sepesi B, Watson TJ, Zhou D, et al. Are endoscopic therapies appropriate for superficial submucosal esophageal adenocarcinoma? An analysis of esophagectomy specimens. J Am Coll Surg 210: 418-427, 2010

21. Lee L, Ronellenfitsch U, Hofstetter WL, et al. Predicting lymph node metastases in early esophageal adenocarcinoma using a simple scoring system. J Am Coll Surg 217: 191-199, 2013.

The Internal Medicine is an Open Access journal distributed under the Creative Commons Attribution-NonCommercial-NoDerivatives 4.0 International License. To view the details of this license, please visit (https://creativecommons.org/licenses/ by-nc-nd/4.0/).

(C) 2019 The Japanese Society of Internal Medicine Intern Med 58: 2467-2472, 2019 